\title{
Flow cytometry to identify leukocyte sub- populations in blood and induced sputum in asthmatic and healthy volunteers exposed to diesel exhaust
}

\author{
Mandy Pui ${ }^{1 *}$, John C Lay ${ }^{2}$, Neil E Alexis ${ }^{2}$, Christopher Carlsten ${ }^{1}$ \\ From AllerGen NCE Inc.'s Fifth Annual Research Conference: Innovation from Cell to Society \\ Québec City, QC, Canada. 7-9 February 2010
}

\section{Objective}

To identify five leukocyte types (in blood and induced sputum) and bronchial epithelial cells (in sputum only) using multi-colour flow cytometry in healthy and mildly asthmatic volunteers exposed to diesel exhaust.

\section{Methods}

Mild asthmatics and normal controls were recruited as study subjects. This crossover study was double-blinded, randomized and counter-balanced to the order of three conditions: diesel exhaust with anti-oxidant, diesel exhaust with placebo, or filtered air with placebo. The subjects were exposed to either filtered air or diesel exhaust (300 ug $\mathrm{PM}_{2.5} / \mathrm{m}^{3}$ ) in a state-of-the-art diesel exhaust exposure facility. An anti-oxidant, $\mathrm{N}$-acetylcysteine $(600 \mathrm{mg})$, or a placebo was taken orally for five days preceding, and on the day of the exposure. Each subject was exposed to each of the three conditions. Peripheral blood samples were taken pre-exposure, and also at 2, 6, and 30 hours after the beginning of exposure. Sputum induction was performed by inhalation of hypertonic saline according to ATS guidelines pre-exposure, and also at 6 , and 30 hours after the beginning of exposure. FACSCanto II (BD Biosciences) was used for flow cytometry. A 5-colour, 12-marker (CD3/CD9/ CD14/CD16/CD19/CD20/CD45/CD56/CD83/CD206/ CD326/HLA-DR) combination was used to identify dendritic cells, macrophages, monocytes, neutrophils, eosinophils, and bronchial epithelial cells. Direct immunolabelling was performed on whole peripheral

${ }^{1}$ University of British Columbia, Vancouver, Canada

Full list of author information is available at the end of the article blood. After incubation, red blood cells were lysed. Remaining cells were washed and resuspended in PBS with $0.5 \%$ paraformaldehyde. Sputum plugs were homogenized with $0.1 \%$ DTT, filtered, and then centrifuged to remove supernatant. Sputum cells were resuspended in PBS at 1 million per $\mathrm{mL}$. Direct immunolabelling was performed. After incubation, cells were washed and resuspended in PBS with $0.5 \%$ paraformaldehyde. Spectral compensation for flow cytometry was performed using an automatic calibration technique (BD CompBeads). Cellular debris was eliminated on the SSC/FSC scattergram. A gating strategy was designed to identify the leukocyte sub-populations and bronchial epithelial cells. Surface markers were chosen based on differential cell-specific expression according to existing literature.

\section{Findings}

The CD45 marker is expressed on all leukocytes. Each cell type of interest has unique scattergram (SSC/FSC) characteristics and/or CD45 expression levels, which are similar but not identical in blood and in sputum. Each cell type has a unique expression pattern of surface markers in blood and in sputum. For example, eosinophils express CD9 whereas neutrophils do not. Findings for the cellular effects of diesel exhaust and anti-oxidants are pending.

\section{Deliverables}

Performing white blood cell differential by standard cytology is common but is poorly reproducible, and labour-intensive. Flow cytometry is superior to standard cytology in identifying rare cells, assessing expression of surface markers, and being automated for quality 
control and efficiency. Multi-colour flow cytometry has previously been employed to identify leukocyte subpopulations in blood with some success, but without a well-standardized strategy. Flow cytometry has been used to identify lymphocytes, and to a lesser extent, phagocytes in sputum, but has rarely been used to identify the rarer sub-populations such as dendritic cells, eosinophils, and bronchial epithelial cells. The findings of this study suggest that a standardized strategy can be created to identify bronchial epithelial cells in sputum, as well as leukocyte sub-populations in blood and in sputum.

\section{Relevance}

Efforts to understand mechanisms of health effects due to ambient air pollution, in order to develop remediation strategies to protect exposed populations (for example, anti-oxidants), are dependent on high-quality and efficient techniques for characterizing cellular effects in the intact human model. Refining the methods described above allows for such detailed assessment of blood and sputum in the context of a controlled human exposure to diesel exhaust.

\section{Author details}

${ }^{1}$ University of British Columbia, Vancouver, Canada. ${ }^{2}$ University of North Carolina, Chapel Hill, USA.

Published: 26 November 2010 